\title{
Differentiation of acute and chronic myocardial infarction using T2-weighted imaging, late enhancement and T1 and T2 mapping - a pilot study at $3 \mathrm{~T}$
}

Florian von Knobelsdorff ${ }^{1,2^{*}}$, Marcel Prothmann ${ }^{1,2}$, Matthias A Dieringer ${ }^{1,2}$, Ralf Wassmuth ${ }^{1,2}$, André Rudolph ${ }^{1,2}$, Wolfgang Utz ${ }^{1,2}$, Julius Traber ${ }^{1,2}$, Andreas Greiser ${ }^{3}$, Thoralf Niendorf², Jeanette Schulz-Menger ${ }^{1,2}$

From 17th Annual SCMR Scientific Sessions

New Orleans, LA, USA. 16-19 January 2014

\section{Background}

Qualitative assessment of myocardial T2-weighted and late enhancement (LGE) images has been demonstrated to differentiate acute from chronic myocardial infarction (AMI, CMI). Parametric mapping could help to overcome challenges in image quality and could contribute to making contrast media application obsolete. The aim of this pilot study was to analyze, whether T2- and T1maps are useful to discriminate AMI from CMI.

\section{Methods}

Eight male patients with acute ST-elevation myocardial infarction underwent CMR at 3T during acute presentation and after $>3$ months latency. Five independent experienced readers, blinded to the patients' clinical state, qualitatively assessed the presence (yes/no) of an infarct-like myocardial lesion in three short axes acquired with several techniques: i) T2-weighted STIR (short-TI triple-inversion recovery prepared fast spin echo), ii) T2-map based on 3 single-shot SSFP (steady state free precession) images with different T2-preparation times, iii) native T1-map based on modified LookLocker inversion recovery using 11 single-shot SSFP images, iv) T1-map 10 minutes after $0.2 \mathrm{mmol} / \mathrm{kg}$ body weight gadobutrol, and v) PSIR (phase sensitive inversion recovery) LGE. The results of all readers were pooled and the sensitivity to determine AMI and CMI was calculated.

\section{Results}

Five readers made a total of 400 decisions (16 CMR exams, 5 different CMR techniques). STIR images were rated as non-diagnostic in 9 decisions, T2-map in 1, native T1-map in 2, T1-map post-contrast in 3. In the remaining image sets, STIR showed an infarct-like lesion in $82.9 \%$ of AMI and $27.8 \%$ of CMI, LGE in $95 \% / 100 \%$, T2-map in $69.2 \% / 35.0 \%$, native T1-map in $86.8 \% / 57.5 \%$, and postcontrast T1-map in $95.0 \% / 91.9 \%$. The combination of STIR and LGE was positive in $77.2 \%$ (AMI) and $27.8 \%$ (CMI), while the combination of maps did not improve the discrimination (table 1). Assuming that STIR, T2-map and native T1-map only depict acute lesions, their specificity to detect AMI was $70.0 \%, 65.0 \%$ and $42.5 \%$.

Table 1 Frequency of an infarct-like lesion in AMI and CMI detected by various CMR techniques

\begin{tabular}{ccc}
\hline Technique & AMI & CMI \\
\hline STIR T2 weighted & 82.9 & 27.8 \\
\hline PSIR LGE & 95.0 & 100.0 \\
\hline T2 Map & 69.2 & 35.0 \\
\hline T1 Map native & 86.8 & 57.5 \\
\hline T1 Map post & 95.0 & 91.9 \\
\hline STIR + PSIR LGE & 77.1 & 27.8 \\
\hline T2 Map + T1 Map native & 62.2 & 32.5 \\
\hline T2 Map + T1 Map post-contrast & 69.2 & 37.8 \\
\hline
\end{tabular}

${ }^{1}$ Cardiac MRI, Charité Medical Faculty, ECRC, Berlin, Germany

Full list of author information is available at the end of the article 


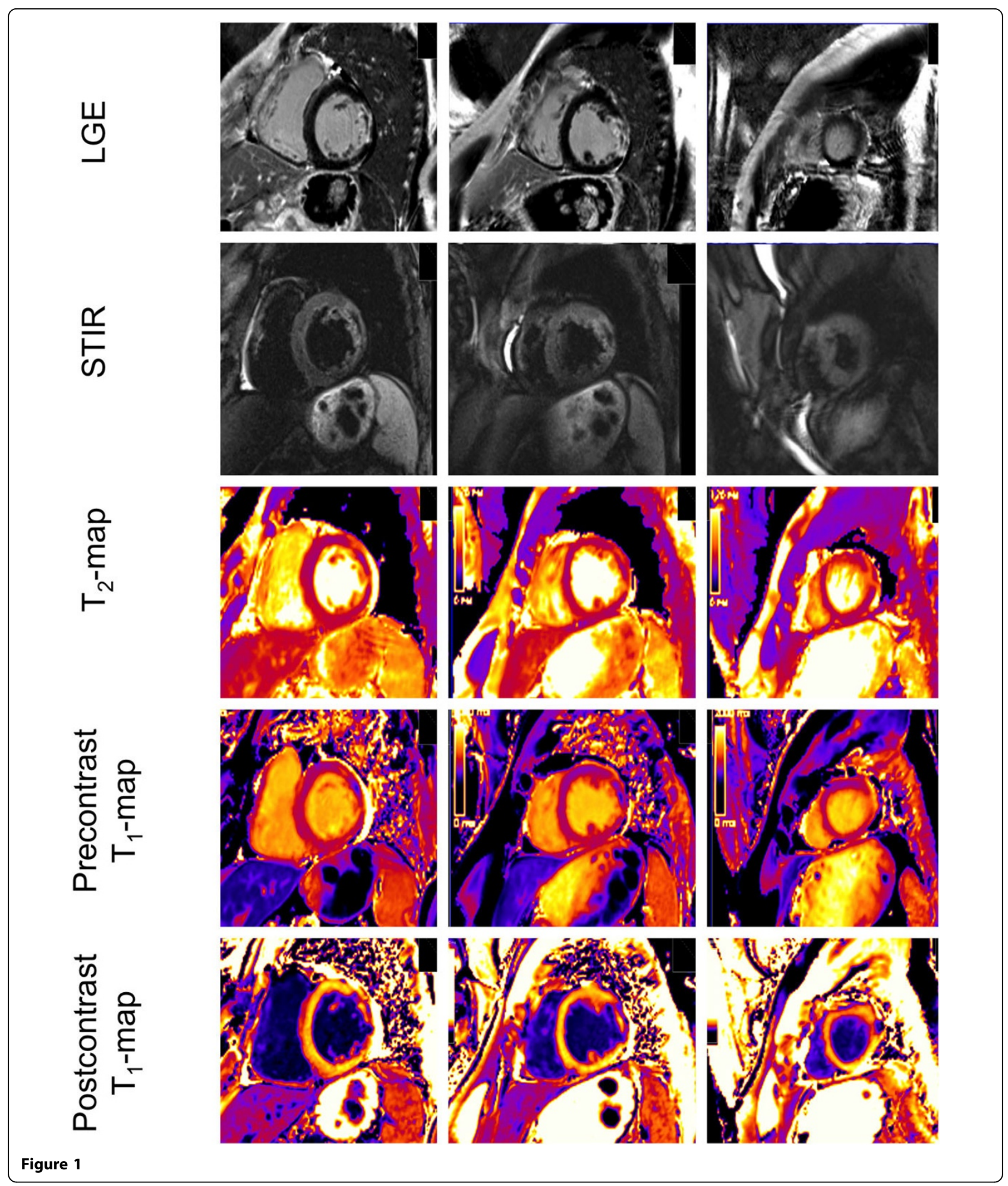

\section{Conclusions}

Post-contrast T1-maps and LGE agree closely in the detection of infarct-like lesions. STIR with diagnostic image quality is superior to detect AMI compared to
T2-mapping, whereas native T1-mapping detects AMI with similar sensitivity as STIR, but with poor specificity. In summary, qualitative assessment of T1- and T2-maps performs not superiorly in the differentiation of AMI 
and CMI compared to STIR and LGE. Further studies are needed that analyze whether quantitative T1- and T2-relaxation times are helpful.

\section{Funding}

Else-Kröner-Fresenius-Stiftung, Bad Homburg, Germany.

\section{Authors' details}

${ }^{1}$ Cardiac MRI, Charité Medical Faculty, ECRC, Berlin, Germany. ${ }^{2}$ Berlin Ultrahighfield Facility, Max-Delbrueck-Center, Berlin, Germany. ${ }^{3}$ Siemens

Healthcare, Erlangen, Germany.

Published: 16 January 2014

doi:10.1186/1532-429X-16-S1-P222

Cite this article as: von Knobelsdorff et al: Differentiation of acute and chronic myocardial infarction using T2-weighted imaging, late

enhancement and T1 and T2 mapping - a pilot study at 3T. Journal of Cardiovascular Magnetic Resonance 2014 16(Suppl 1):P222.

Submit your next manuscript to BioMed Central and take full advantage of:

- Convenient online submission

- Thorough peer review

- No space constraints or color figure charges

- Immediate publication on acceptance

- Inclusion in PubMed, CAS, Scopus and Google Scholar

- Research which is freely available for redistribution

Submit your manuscript at www.biomedcentral.com/submit 